P-266 DESIGNING A FRAMEWORK TO PROACTIVELY SUPPORT NURSES THROUGH THEIR REVALIDATION PROCESS, ENSURING EXCELLENT AND PROFESSIONAL STANDARDS OF CARE ARE MAINTAINED

Leigh Marrs. St Oswald's Hospice, Newcastle upon Tyne, UK

10.1136/bmjspcare-2016-001245.285

Background A hospice has been preparing for nurse revalidation, ensuring staff feel fully supported and valued so that the best, safest care is delivered to patients and families.

Following on from the Francis Report recommendations, the Nursing and Midwifery Council (NMC) concluded that nurses are to be revalidated every three years to maintain their registration.

The hospice has fully trained and supported staff in how to undertake the process, with four members of staff currently applying for their revalidation. This involves completing their portfolio, which contains records of their CPD, practice hours, feedback and reflective accounts.

Although the recommendation is only for registered nurses and midwives, the organisation has extended this and included auxiliary nurses and professionals allied to medicine (PAMs) in this process. It was decided that this would help set a consistent approach in terms of establishing reflective practice across the workforce, creating a professional standard for all clinical staff involved in patient care.

Aim The purpose of revalidation is to improve public protection by making sure that nurses continue to practice safely throughout their career.

The aim of the project was to ensure that the organisation and its staff were prepared for revalidation. This meant looking at all their systems and ensuring that they were aligned to support nurses with the requirements of revalidation. By doing this the hospice has been able to redesign appraisal and induction, promote educational opportunities and strengthen the support available to nurses and other staff.

Method The hospice conducted training sessions that briefed staff on the requirements of revalidation, signposted the key requirements and provided guidance on how to build their portfolio.

In addition, the clinical education nurse has provided one-toone support to people who were unable to attend the workshops or required further support, meaning that to date a total of $90 \%$ of nurses have benefited from this initiative.

The hospice has a designated administrator for the project and has trained line managers of nurses in the Confirmer role.

Conclusion Once the first wave of nurses has completed the revalidation process, the organisation will review the work to date and create a framework to continue to support its staff with revalidation.

\section{P-267 A UNIQUE CAREER PATHWAY FOR HCAS IN A HOSPICE SETTING}

Debra Broadhurst, Donna Walker, Mandy Motley. LOROS Hospice, Leicester, UK

\subsection{6/bmjspcare-2016-001245.286}

Background The Willis Commission advocated a career pathway for health care assistants (HCAs) to enhance career opportunities and support the sustainability of the future nursing workforce. Several opportunities have been embraced to enable this including the creation of assistant practitioner (AP) roles, health and social care apprenticeships alongside, underpinning education such as Foundation Degrees, QCF frameworks and the Care Certificate.

Our Experience For the past nine years our Education Department has run a Foundation Degree which several HCAs from the hospice have successfully achieved. More recently, engagement in level 2 and 3 QCF modules has taken place. In addition, there has been a variety of activity to support HCA development in a hospice setting:

- Two assistant practitioner posts (in Education \& MND Team)

- Four trainee assistant practitioners - rotating into the community trust as part of collaborative pilot project

- Two clinical apprenticeships (inpatient ward and day therapy rotational posts)

- Two HCA development posts, through 'talent managing' staff from the domestic team \& HCA bank

- Three senior HCA posts on the inpatient ward.

The above initiatives have demonstrated the commitment of the organisation to develop our workforce and embed novel roles within the nursing teams in alignment with national directives. The Education AP has developed and embedded the care certificate with new HCAs. One of the clinical apprentices was previously a volunteer and the three senior HCAs have been internally promoted. The HCA development posts were created to enable appropriate staff who lacked traditional essential application criteria, to learn on the job, whilst undertaking supported study to attain vocational qualifications. Both clinical apprentices have successfully attained a substantive post at the end of their training; the success of this means we are seeking to appoint a further two clinical apprentices this year.

\section{P-268 DIVERSITY CLIMATE SURVEY IN A HOSPICE SETTING}

Susan Bridger. Farleigh Hospice, Chelmsford, UK

\subsection{6/bmispcare-2016-001245.287}

Diversity' refers to all significant differences between individuals including sex, age, race, ethnicity, religion, physical and mental ability, educational achievement and occupation.

In 2013, as part of the Equality and Diversity work plan it was decided to survey staff's views and perceptions of equality and diversity in the hospice. It was agreed to use a standardised tool Diversity Climate Survey - developed by Prof Robert Bean, an Australian business consultant and with his agreement some questions were slightly modified to reflect ethnicity and religion relevant to the UK.

The Diversity Climate Survey is intended for use across an entire workforce and provides data on individual, work group and organisational factors relating to the ways individuals perceive and feel about the presence and management of diversity in their workplaces and the influences that aspects of diversity and diversity management may have on their career development. The results of the survey can evidence themes around values and belief providing a baseline to target future training and policy in relation to issues of equality and diversity

Farleigh Hospice scoring was positive achieving Level 4 with a score of 60.97 out of 69 . and demonstrated that respondents felt the hospice was managing diversity well and they felt positive about workforce diversity. To improve scores the aim would be to target questions which scored the lowest and these would identify also if the areas needing attention related to individual, 
department or organisational issues. An action plan was develop to address the lowest scoring areas.

The survey was completed again in 2015 demonstrating similar positive results achieving Level 4 again with a score of 61.05 out of 69 .

\section{P-269 EMBRACING CHANGE: FOCUSING ON OUR PEOPLE, OUR PARTNERSHIPS AND OUR POTENTIAL, ARTHUR RANK HOSPICE CHARITY: PART 2 OF OUR JOURNEY}

Liz Webb. Arthur Rank Hospice Charity, Cambridge, UK

\subsection{6/bmjspcare-2016-001245.288}

Arthur Rank Hospice Charity is on a dual journey of moving from the NHS to independence and building and moving to a new hospice building. This presented us with a double dose of significant change for our staff in the space of 18 months.

We recognised that we have an opportunity to shape and transform our organisational culture as a new team- a mixture 100 plus NHS staff and 20 previous charity staff. At the same time as embedding the NICE pathway-Workplace health policy and management practices (2015). Managing the changes taking place with our staff is vital to the ongoing success of the charity and quality of care that we provide.

What we did

Pre TUPE of 100 NHS staff

- Charity CEO and NHS Clinical lead worked together to communicate clearly and robustly with both teams about what was happening and when.

- Occupational health support and 24/7 access to counselling.

\section{At point of integration of the two previous teams}

- Effective communication. Programme of face to face meetings with the new senior leadership team:

- Communicated a new quality governance structure that supports information flow from bottom to top of the organisation.

- Commissioned a series of cultural workshops with all staff over 10 weeks. All staff were invited to the full day events and all attended except three.

\section{Outcomes of workshops and next steps}

Using a model of 'appreciative enquiry' the workshops identified where we were and what steps we needed to take to embed a culture of openness, transparency, innovation and change.

A plan of work was developed to support the new culture and focus on wellbeing with the now integrated staff team.

This includes

- Fortnightly bulletin

- Staff Forum

- clinical supervision for all clinical staff.

- 1.1 meetings with staff and line manager

- annual appraisal and clear objectives

- Research based journal club

- Closing the loop', ensuring information and outcomes from projects, investigations and complaints and shared with all.

- Move to new building-program of includes a team approach to resilience and change management.
P-270 THE MACMILLAN VALUE BASED STANDARDS: HOW A HOSPICE TOOK MACMILLAN VALUES TO ITS HEART

Sandie Robinson, Roger Wheelwright. John Taylor Hospice, Birmingham, UK

10.1136/bmjspcare-2016-001245.289

How a hospice adopted a culture of care by using Macmillan Values Based Standard (MVBS) where a patient and their loved ones are respected and valued. Fostering positive attitudes with staff to help shape services and reflect on the quality of their care.

MVBS were developed to bridge the gap between the principles associated with person-centred care and the behaviours and expectations that should form part of healthcare practices.

The eight standards set out behaviours with front line staff which are at the centre of driving forward standards of service. Staff awareness of the MVBS, allowing ideas and innovations to improve care from the bottom up. Staff engagement is essential to allow change and improvement to care provision and sustainability of a quality service. Clinical supervision of staff, enables teams to analyse situations and suggest different ways of working. The reflection process enables conceptualisation of how they can integrate the fundamental values and behaviours set out in the MVBS within their everyday practice.

Team meetings recap on standards focussing on one standard each month to remind staff how the standard can be implemented into their working day. Feedback from service user experience is at the heart of what we do and essential to evaluate care and prevent disconnection. One main measure from any individual within a hospice organisation is to question 'have I made a difference to that individual?" "How we care for the dying is an indicator of how we care for all sick and vulnerable people".

Using the MVBS to set our standards of care as an organisation allows our staff to measure themselves individually and as a team. Through processes of reflection, clinical supervision, peer support and real time feedback we can continue to strive for excellence in care and influence change and improvement to our service.

\section{P-271 OPTIMISING TEAM WORKING IN WIGAN \& LEIGH HOSPICE NURSE SPECIALIST SERVICE}

Jenny Gallagher, Rebecca Lennon. Wigan and Leigh Hospice, Wigan, UK

\subsection{6/bmjspcare-2016-001245.290}

Introduction Effective teams improve quality, whilst reducing staff stress levels. The degree of self-rated teamwork is also related to patient and carer satisfaction. Teams do not work just because they exist and conflict is both inevitable and healthy. We undertook a review of our own team working strategies with the aim to improve collaborative working, inter and intra-team communication, time management and understand the diversity within our team. Overall, we aimed to uncover potential and identify opportunities to develop.

What did we do?

- Completed an anonymised survey evaluating how the team currently functions.

- Developed a purpose statement and operating principles.

- Team service objectives were reviewed.

- Time management training. 